\title{
Construção do acolhimento com classificação de risco em uma Unidade Básica de Saúde
}

Tainá de Jesus Alves Portela ${ }^{1}$, Quiteria Larissa Teodoro Farias ${ }^{2}$, Maristela Inês Osawa Vasconcelos ${ }^{3}$

\section{Resumo}

O estudo tem como objetivo descrever a experiência de acadêmicos de enfermagem no desenvolvimento do Acolhimento com Classificação de Risco para profissionais e usuários de uma Unidade Básica de Saúde. Trata-se de um relato de experiência que traz a ação desenvolvida por acadêmicos do $8^{\circ}$ semestre do Módulo de Internato 1 do Curso de Graduação em Enfermagem da Universidade Estadual Vale do Acaraú na implantação do Acolhimento com Classificação de Risco em uma Unidade Básica de Saúde da região noroeste do estado do Ceará. As ações foram estruturadas em 6 encontros, nos quais dois envolveram os profissionais da equipe de enfermagem, um englobou todos os trabalhadores da unidade básica e três abordaram os usuários do serviço a partir do qual temáticas e materiais estudados anteriormente possibilitaram a construção de dispositivos facilitadores do aprendizado dos participantes. Os resultados desse estudo são apresentados e discutidos em dois tópicos essenciais para a elaboração das metodologias: organização do trabalho e transformação das práticas profissionais; e potencialização no atendimento da/para população. As estratégias de educação permanente com os profissionais da unidade e educação em saúde com os usuários adotadas na experiência constituíram-se como potencializadoras na transformação das práticas do trabalho.

\section{Palavras-chaves}

Acolhimento. Atenção Primária à Saúde. Educação continuada. Educação em saúde. Demandas de serviços de saúde.

1 Graduada em Enfermagem pela Universidade Estadual Vale do Acaraú, Ceará, Brasil; residente multiprofissional em Neonatologia pela Santa Casa de Misericórdia de Sobral/Centro Universitário Uninta; membro do Grupo de Pesquisa e Estudo em Vulnerabilidades e Saúde (GEVS/UVA). E-mail: tainadejesusalves@gmail.com.

2 Mestra em Saúde da Família pela Universidade Federal do Ceará, Brasil; membro do Laboratório de Pesquisa Social, Educação Transformadora e Saúde Coletiva (LABSUS/UVA). E-mail: larissa.teodoro1996@gmail.com.

${ }^{3}$ Doutora em Enfermagem pela Universidade Federal do Ceará, Brasil; estágio pós-doutoral na Universidade Estadual do Ceará, Brasil; professora na Universidade Estadual Vale do Acaraú, Ceará, Brasil; líder do Laboratório de Pesquisa Social, Educação Transformadora e Saúde Coletiva (LABSUS/UVA). E-mail: miosawa@gmail.com. 


\title{
Development of reception with risk classification at a Basic Health Unit
}

Tainá de Jesus Alves Portela ${ }^{4}$, Quiteria Larissa Teodoro Farias ${ }^{5}$, Maristela Inês Osawa Vasconcelos ${ }^{6}$

\begin{abstract}
The aim of this study is to describe the experience of nursing school students in the development of Reception with Risk Classification for professionals and users of a Basic Health Unit. The paper is an experience report that shows the action developed by 8th semester students in the Internship I module of Vale do Acaraú State University nursing major, in the implementation of Reception with Risk Classification at a Basic Health Unit in northwestern Ceará. The actions were organized in six meetings, two of them assembled the professionals of the nursing team, one encompassed all the staff of the Basic Health Unit, and three brought together the unit service users. Based on subjects and materials previously studied, the meetings allowed for the construction of devices that facilitate the participants learning. The results of the study are presented and discussed in two essential topics for the development of methodologies: work organization and transformation of professional practices; and optimization of the service to/for the population. The strategies of permanent education adopted in the experience with the professionals of the unit and of health education, as well as the unit users can be regarded as an enhancer in the transformation of work practices.
\end{abstract}

\section{Keywords}

User embracement. Primary Health Care. Education continuing. Health Education. Health services demand.

\footnotetext{
${ }^{4}$ Graduated in Nursing, Vale do Acaraú State University, State of Ceará, Brazil; multidisciplinary resident in Neonatology at the Santa Casa de Misericórdia de Sobral/Uninta University Center; member of the Research and Study Group on Vulnerabilities and Health (GEVS/UVA). E-mail: tainadejesusalves@gmail.com.

${ }^{5}$ Master in Family Health, Federal University of Ceará, State of Ceará, Brazil; member of the Laboratory for Social Research, Transformative Education and Collective Health (LABSUS/UVA). E-mail: larissa.teodoro1996@gmail.com.

${ }^{6} \mathrm{PhD}$ in Nursing, Federal University of Ceará, State of Ceará, Brazil; post-doctoral internship at the State University of Ceará, State of Ceará, Brazil; professor at Vale do Acaraú State University, State of Ceará, Brazil; leader of the Laboratory for Social Research, Transformative Education and Collective Health (LABSUS/UVA). E-mail: miosawa@gmail.com.
} 


\section{Introdução}

A Atenção Primária à Saúde (APS) é, preferencialmente, o primeiro contato da população ao serviço de saúde, oferecendo atendimento abrangente, acessível e baseado na comunidade, com projeção de resolutividade de 80 a $90 \%$ das necessidades de saúde da população ao longo da vida (OPAS, 2019).

A Portaria $n^{\circ} 2.436 / 2017$ salienta que a Estratégia Saúde da Família (ESF), enquanto APS brasileira, é a ordenadora do cuidado dentro das Redes de Atenção à Saúde (RAS) e prioritária para consolidação e expansão da atenção primária, proporcionando um acesso universal e um acolhimento baseado na real necessidade do usuário, família e comunidade (BRASIL, 2017). Sendo assim, responsável pelo fluxo do usuário na RAS, estruturada na interação de três elementos constitutivos: uma população específica, uma estrutura operacional e um modelo de atenção à saúde (BRASIL, 2015).

Para a efetividade dessa organização, o Ministério da Saúde (MS) adotou o Acolhimento com Classificação de Risco nas Unidades Básicas de Saúde (UBS). Para tanto, determina como forma de classificar e avaliar as vulnerabilidades da população que procura atendimento em busca da promoção de equidade e fomento para a implantação de uma boa prática clínica. A troca de conhecimentos e informações entre a equipe multiprofissional permite um entendimento e acolhimento das mazelas territoriais potencializando intervenções das diferentes categorias assistenciais no cuidado à demanda espontânea (BRASIL, 2013).

Entre as tecnologias utilizadas para reorganização dos processos de trabalho, o Acolhimento com Classificação de Risco se corrobora como um mecanismo enérgico que propicia resultados relacionados a maior satisfação de usuários e trabalhadores, aumento do êxito na parte clínica dos serviços e um desencadeante de demais mudanças, como a composição das equipes de referência, coparticipação na gestão, a consolidação/fortificação das RAS e o enaltecimento com a apreciação do trabalho em saúde (BRASIL, 2020). Porém, sua implantação ainda é algo gradativo dentro do serviço da ESF. Logo, assim como todos os avanços no serviço, faz-se necessária uma ressignificação das práticas dos profissionais que atuam, bem como da própria população atendida pelo serviço. Para isso, o Ministério da Saúde traz a Política Nacional de Educação Permanente em Saúde (PNEPS) como uma potente ferramenta de aprendizado no e para o serviço, de forma a gerar observações sobre o processo de trabalho dos profissionais, autogestão, mudança institucional e mudanças nas 
práticas assistenciais, por meio do aprender, do trabalho em equipe, de estabelecer a construção de rotinas e aprendizagem individual, coletiva e institucional (BRASIL, 2017).

Para compartilhar essas mudanças com a população de forma didática e facilitar a aproximação entre a comunidade e o serviço, o uso da sala de espera é indicado na literatura como uma estratégia potente realizados em serviços da atenção básica para o desenvolvimento de ações orientadas ao acolhimento, prevenção de doenças, promoção e educação em saúde (SILVA et al., 2018; SILVA et al., 2019).

Dessa forma, a utilização das práticas educativas no processo de Educação Permanente em Saúde (EPS) e Educação em Saúde possibilitam uma integração entre teoria e prática, fomentando uma formação mais sólida, compreensível e efetiva que, ao relacionar-se com a realidade, possibilita o entendimento e prática das tecnologias de saúde criadas.

No tocante, a EPS estimula o desenvolvimento do pensamento crítico numa maior conscientização dos fenômenos sociais e resolubilidade de problemas na reorganização dos processos de trabalho por meio do Acolhimento com Classificação de Risco. Essa implementação efetiva o princípio de equidade e emprega estratégias que garantem a atenção à demanda espontânea, o que permite ao enfermeiro utilizar seus conhecimentos técnicocientíficos na assistência, garantindo um cuidado único, continuado e qualificado nos serviços de saúde, inclusive na ESF (BUSNELLO et al., 2017).

O presente estudo possibilita, portanto, o compartilhamento de experiências de EPS e Educação em Saúde para organização e reordenação dos fluxos de pacientes de uma UBS do interior do Ceará, tornando-se relevante, à medida que servirá de subsídio para a adoção das estratégias utilizadas em outras unidades.

Dessa forma, o estudo tem como objetivo descrever a experiência de acadêmicos de enfermagem no processo de implantação do Acolhimento com Classificação de Risco em uma UBS.

\section{Metodologia}

O estudo baseia-se em um relato de experiência que traz a ação de acadêmicos de enfermagem do $8^{\circ}$ semestre de graduação no desenvolvimento do Acolhimento com Classificação de Risco em uma UBS da região noroeste do estado do Ceará, correspondente a carga horária de ensino-extensão do Módulo de Internato I da Universidade Estadual Vale do Acaraú (UVA). 
Segundo Lima e Cavalcante (2012), os relatos de experiência são uma ferramenta de pesquisa descritiva que apresentam uma reflexão sobre uma ação ou um conjunto de ações que abordam uma situação vivenciada de interesse da comunidade científica.

Optou-se por transformar a experiência em narrativa, salvo o impacto da mesma para a reorganização dos serviços na APS com objetivo de beneficiar acadêmicos/profissionais e ambientes de ensino/saúde na qualificação do processo de trabalho da ESF, atualizando antigas práticas e modelando novas perspectivas para concretização dos princípios do SUS.

A UVA propicia ao futuro enfermeiro, nos últimos três semestres de graduação, estar em contato integral com a assistência nos seus três níveis: Atenção Primária, Secundária e Terciária. Portanto, o Internato I leva o estudante ao encontro das práticas profissionais da APS.

A imersão ocorreu durante o período de três meses, de maio a agosto de 2019. Participaram do estudo junto aos acadêmicos, a equipe de enfermagem da ESF; a população adscrita no território da UBS; e os demais profissionais responsáveis pela organização da unidade. Foram realizados seis encontros para o desenvolvimento das atividades, a divisão deles pode ser observada no quadro 1 .

Quadro 1 - Encontros, temáticas, metodologias, materiais e participantes dos encontros realizados na UBS cearense, 2019

\begin{tabular}{|c|c|c|c|c|}
\hline Encontros & Temática & Metodologia & Materiais & Participantes \\
\hline \multirow[t]{2}{*}{$1^{\circ}$} & $\begin{array}{l}\text { A essência do } \\
\text { acolhimento na APS }\end{array}$ & $\begin{array}{l}\text { Mural do } \\
\text { Acolhimento } \\
\text { (Mapa Conceitual) }\end{array}$ & Tarjetas e pincéis & \multirow{2}{*}{$\begin{array}{l}5 \text { Enfermeiros da } \\
\text { UBS ( } 3 \\
\text { assistenciais, uma } \\
\text { residente e a } \\
\text { gerente) }\end{array}$} \\
\hline & $\begin{array}{l}\text { Desmitificando a } \\
\text { Classificação de Risco } \\
\text { na prática de } \\
\text { enfermagem }\end{array}$ & $\begin{array}{l}\text { Caminho da } \\
\text { Classificação }\end{array}$ & & \\
\hline \multirow[t]{2}{*}{$2^{\circ}$} & $\begin{array}{l}\text { Teorias e práticas: } \\
\text { Acolhimento com } \\
\text { Classificação de Risco } \\
\text { na UBS. }\end{array}$ & Estudo de caso & $\begin{array}{l}\text { Papéis, pincéis e } \\
\text { tarjetas }\end{array}$ & \multirow[t]{2}{*}{$\begin{array}{l}5 \text { Técnicos de } \\
\text { Enfermagem ( } 4 \\
\text { assistenciais e } 1 \text { da } \\
\text { Sala de Vacina) }\end{array}$} \\
\hline & $\begin{array}{l}\text { Acolhimento com } \\
\text { Classificação de Risco: } \\
\text { como proceder? }\end{array}$ & $\begin{array}{l}\text { Caminho da } \\
\text { Classificação }\end{array}$ & & \\
\hline $3^{\circ}, 4^{\circ}$ e $5^{\circ}$ & $\begin{array}{l}\text { Acolhimento com } \\
\text { Classificação de Risco }\end{array}$ & Salas de espera & $\begin{array}{l}\text { Slide, data show, } \\
\text { fita adesiva e } \\
\text { pincéis }\end{array}$ & $\begin{array}{l}\text { População adscrita } \\
\text { do território }\end{array}$ \\
\hline
\end{tabular}




\begin{tabular}{|c|c|c|c|c|}
\hline & $\begin{array}{l}\text { Organização do fluxo } \\
\text { da UBS }\end{array}$ & $\begin{array}{l}\text { Salas de espera e } \\
\text { informativos: } \\
\text { aplicação da } \\
\text { tecnologia de } \\
\text { educação em saúde }\end{array}$ & & \\
\hline Adicional & $\begin{array}{l}\text { Cuidar de quem cuida: } \\
\text { um gesto de } \\
\text { agradecimento e amor }\end{array}$ & $\begin{array}{l}\text { Corredor do } \\
\text { cuidado } \\
\text { pProfissional }\end{array}$ & $\begin{array}{l}\text { Óleos, hidratante, } \\
\text { ambiente } \\
\text { climatizado, } \\
\text { bacia, bolas, } \\
\text { TNT, perfume, } \\
\text { espelho }\end{array}$ & $\begin{array}{l}\text { Profissionais em } \\
\text { geral presentes na } \\
\text { UBS }\end{array}$ \\
\hline
\end{tabular}

Fonte: Os autores (2020).

O primeiro encontro permitiu a análise de conhecimento dos profissionais sobre os métodos de acolher a população, por meio de quatro perguntas-chaves: "o que é?", "o que necessita?", "quem realiza?" e "como fazer?", que culminou na elaboração do Mural do Acolhimento, fixado na sala de reuniões. Todos puderam apresentar sua visão sobre o assunto e discutir as necessidades da UBS referentes à temática, levando à reflexão sobre o ambiente de trabalho.

O segundo encontro baseou-se em estudos de caso que levaram aos participantes refletir na resolução da demanda apresentada, quais os sinais vitais avaliados, assim como sua possível classificação se fosse remanejada para a intervenção de enfermagem.

$\mathrm{Na}$ segunda fase do $1^{\circ}$ e $2^{\circ}$ encontro, pôde-se trilhar o Caminho da Classificação composto por tarjetas classificatórias: Emergente, Urgente, Pouco Urgente e Agendado; além de Targetas Problemáticas com casos frequentes das unidades de saúde e que eram classificados de forma errônea, prejudicando o fluxo de atendimento e triagem. Foi elaborada assim, uma nova classificação de risco para a unidade baseada no referencial do Ministério da Saúde, que se baseia nas cores azul para situações não agudas passíveis de orientações ou agendamentos; verde para atendimentos do dia com risco baixo ou ausência de risco, com vulnerabilidade importante; amarelo para atendimentos prioritários com risco moderado; e vermelho para atendimento imediato com risco de vida (BRASIL, 2013). Mediante tal afirmativa, destaca-se o Acolhimento com Classificação de Risco como um dinamismo fortalecedor da cooperação entre as equipes a fim de adaptar a ESF à demanda espontânea e permitir o cuidado integral e resolutivo.

O $3^{\circ}, 4^{\circ}$ e $5^{\circ}$ encontros voltaram-se para a população atendida e a necessidade de conhecimento dos mesmos sobre o fluxo do atendimento. Realizou-se ainda, momentos como 
Salas de Espera com recursos visuais e verbais para informatização da população sobre as eventuais mudanças a serem realizadas na unidade de saúde.

Por fim, o Corredor do Cuidado Profissional foi uma ferramenta extra utilizada para proporcionar aos profissionais um momento de relaxamento, diante de um ambiente calmo, climatizado, com massagens e frases motivacionais com os profissionais gerais da UBS.

\section{Resultados}

Os resultados desse estudo são apresentados e discutidos em dois tópicos principais pautados na experiência dos acadêmicos, sendo considerados pontos de partida para a elaboração das metodologias.

\section{Organização do trabalho e transformação das práticas profissionais}

Após cerca de dois meses de imersão na UBS, a construção de vínculos com os profissionais possibilitou a realização de encontros com a equipe de enfermagem em duas etapas envolvendo o Acolhimento com Classificação de Risco e sua prática para aprimoramento/agilidade na organização dos fluxos da demanda espontânea. Vale ressaltar que a realização da Classificação de Risco é incumbência da Enfermagem, pois cabe à mesma a realização de técnicas complexas com embasamento científico e tomada de decisões rápidas e resolutivas amparadas na Lei n. ${ }^{\circ} 7.498$ (BRASIL, 1986).

$\mathrm{O}$ atendimento da UBS referida era determinado por agendamento, se retorno para consulta médica, e por meio de fichas referentes à ordem de chegada de cada paciente, urgindo a reordenação dos fluxos e transformação da realidade local, a fim de atender as necessidades do usuário, convertendo a triagem excludente em um modelo acolhedor.

Os primeiros encontros tiveram como foco a equipe de enfermagem tendo em vista que ela tem por fundamento/essência o cuidado ao ser humano, centrado na tríade família, comunidade e indivíduo, construindo ações estruturadas na responsabilização do acolhimento, cuidado, conforto e bem-estar dos usuários (ROSSATO et al., 2018).

Compreende-se que a reorientação da formação profissional em saúde consiste em um dispositivo de fortalecimento do SUS, por conseguinte, a educação em serviço desenvolve função essencial de cooperação na estruturação do novo sistema de saúde. Nesse sentido, o processo de EPS representa um ambiente de debates onde os atores, que são participantes dos 
momentos, possuem uma notoriedade e importância na ressignificação do trabalho em saúde (ALMEIDA et al., 2016).

Dessa forma, todos os momentos foram articulados de forma a favorecer esse processo. No primeiro encontro, a construção do Mural do Acolhimento e as reflexões geradas por ele permitiram uma maior compreensão da assistência num caráter integral e universal aos que buscam atendimento na unidade. Indagar aos profissionais sobre o conhecimento do significado de acolher na APS contribuiu para debate e para a construção coletiva de ideias, embasadas na literatura científica, para implantação na UBS em questão.

A retórica dos profissionais ao expressar, de forma geral em suas tarjetas, que o acolhimento é uma vinculação e constitui papel de todos os profissionais da unidade, por meio da empatia e escuta qualificada, com fins de organizar o serviço e dar direcionamento às necessidades dos usuários, representa o primeiro passo para a modificação de práticas já instituídas no serviço que, por vezes, não favorecem o processo de integralidade e equidade na assistência.

No segundo encontro, os estudos de caso, desenvolvidos com os profissionais técnicos de enfermagem, se tornaram outro ponto essencial como sanador de dúvidas e fortalecedor do aprendizado, padronizando os conhecimentos entre eles e fixando a necessidade da realização da mensuração dos sinais vitais, antes ignorados/não realizados corretamente, aliados à Classificação de Risco, determinante para uma ação conjunta de toda a equipe de enfermagem.

Assim como destacado por Rossato et al. (2018), notou-se a ênfase na necessidade que as equipes de enfermagem, assim como outros profissionais, têm de aderir conhecimentos sobre o acolhimento. $\mathrm{O}$ estudo ainda corrobora situações vivenciadas nessa pesquisa ao comprovar que as dificuldades de entendimento partiram dos técnicos de enfermagem, devido a esses profissionais estarem dentre os primeiros a acolher os usuários, necessitando agir no resgate de competências clínicas para avaliação que por vezes se perdem na rotina do processo de trabalho.

Nos dois encontros, com a construção dos Caminhos da Classificação, houve uma desmistificação e o entendimento claro das propostas do MS acerca da Classificação de Risco na prática da equipe de enfermagem: como proceder frente à dinâmica do serviço, conhecimento do fluxo de atendimento para o trabalho profissional efetivo e satisfação do paciente. 
As metodologias utilizadas favoreceram os sujeitos a refletirem, de modo incessante, sobre a realidade, buscando resoluções criativas para o enfrentamento e superação dos problemas dos serviços de saúde, portanto, qualificando intervenções voltadas para o alcance da resolutividade promovendo a eficácia do sistema de saúde (FRANÇA et al., 2017).

Os momentos foram finalizados com a realização do Corredor do Cuidado com todos os profissionais da UBS, por meio da proposta de acolher o profissional para que ele se torne capaz de acolher a população. O momento foi terapêutico e possibilitou o alívio da tensão, o escape da realidade, o autoconhecimento de suas necessidades e a reflexão sobre o cuidado do outro.

Em uma relação clínica, o Corredor do Cuidado preza o outro como centro de sua prática, em que a esfera designada de "cuidado" envolve esse momento de interação com o próximo, aliado aos elementos que permitem uma aproximação eficaz valorizando a interpretação do processo saúde-adoecimento dos sujeitos. Mediante tal pensamento, o cuidar surge como ponto centralizador nas práticas dos profissionais de saúde, urgindo que todos os trabalhadores da área compreendam os elementos constitutivos desse cuidado para aplicá-los de maneira individual, universal e equitativa (CARNUT, 2017).

Enquanto facilitadores das ações, cabe destacar o desenvolvimento do senso de gerenciamento de cuidados, organização da demanda, cuidado qualificado e trabalho em equipe que foram pontos chave desse processo de aprendizado gerado para a construção do futuro enfermeiro e modificação da realidade dos profissionais já em serviço.

\section{Potencialização no atendimento da/para população}

Segundo Silva et al., (2016), a construção de políticas públicas de saúde, nas últimas décadas, enfatiza a indispensabilidade da implantação de inovadoras práticas de saúde voltadas para a integralidade do cuidado. Nessa perspectiva, o serviço de Acolhimento com Classificação de Risco contribui para o adequado atendimento nas unidades de saúde.

Compreendendo a importância de compartilhar esse processo de construção com os usuários que recebem atendimento no serviço, de forma a incorporar a população nesse processo, utilizou-se os espaços de sala de espera para desenvolver educação em saúde. Uma ferramenta estratégica que permite transformar um espaço, por vezes, de angústia e estresse, em local de fala, escuta e construção de vínculos, de significados, de atenção, de interesse, de 
orientação, de aprendizado e de prazer, que influenciaram para a internalização de conhecimentos (SILVA et al., 2018; NEGRÃO et al., 2018).

Levando em consideração que a implementação dessa nova forma de acolher parte principalmente da queixa da população à demora no atendimento e a preferência pelo horário de chegada na UBS. Assim, tem-se a oportunidade de compreender que o serviço de assistência se estrutura em um processo categorizado por cores, tempo e prioridade de sinais, sintomas ou idade.

Para desenvolver esses momentos, utilizou-se os espaços intra e extra consultórios, para discussão das mudanças e construção de conhecimentos junto ao território. Foram elaborados fluxogramas informativos, de acordo com o preconizado pelo MS, e anexados junto às portas dos consultórios, Serviço de Assistência Municipal Especializada (SAME) e farmácias para facilitar o entendimento do novo fluxo da unidade e estabelecer uma nova visão do serviço desde a chegada até o atendimento conforme a necessidade.

Os momentos permitiram aos sujeitos envolvidos compreender que a pessoa que busca pelo cuidado tem necessidade de assumir o papel ativo nesse processo, de forma a interagir a partir de suas experiências com o que lhe é significativo, ajudando a buscar respostas para as suas necessidades, baseando-se no diálogo com os saberes prévios dos usuários dos serviços de saúde, seus saberes populares e na análise crítica da realidade (NEGRÃO et al., 2018; FALKENBERG et al., 2014).

As tecnologias dinâmicas e de fácil compreensão permitiram o estabelecimento do foco da população ao que era explicitado. O saber dialogar foi essencial para adaptar a linguagem rebuscada a uma popular para que os pacientes pudessem questionar e opinar, assim eles mostraram-se mais críticos e dispostos a colaborar com o novo fluxo do sistema.

A problematização de uma temática que afeta diretamente a comunidade aliada à construção compartilhada do conhecimento e o compromisso de estabelecer um projeto que beneficie o diálogo e direitos democráticos dos pacientes são contextos importantes, fruto das ações realizadas. Permitiu-se a igualdade e universalidade da saúde, protagonizando uma participação popular no cuidado em saúde, na intersetorialidade e na produção de conhecimento integrando educação popular e EPS como modificantes da realidade local.

A troca dos saberes técnico-científicos e populares entre profissionais e usuários, por meio do diálogo, contribuíram na construção de um vínculo que envolve a equipe, o usuário, a família e estende-se à comunidade, constituindo, assim, uma proposta de integralidade (HANAUER; MADUREIRA, 2017). 
A partir disso, houve redução no tempo de espera, diminuição da demanda espontânea, vinculação com a população, informação verbal e não verbal acessível e a construção de usuários como agentes de mudança do território. Destaca-se, aqui, a importância do contato do atual acadêmico em formação com os usuários do serviço, além do posterior incentivo aos atuais profissionais de realizarem a mesma firmação de laços.

Construir uma parceria com a população e, assim, buscar formas de entendê-la, tornase uma ferramenta que age no incentivo de novas e únicas habilidades de cuidado e vínculo ao envolver pontos como a escuta qualificada, tecnologias diversificadas/dinâmicas de educação acessíveis ao entendimento popular, compreensão das necessidades de saúde/pessoais em prol de adaptações do ambiente para despertar o conforto/confiança dos pacientes e estruturação aliada a implantação de novas práticas de trabalho em saúde que visem o próximo ao invés de apenas mecanizar o processo de trabalho.

Acolher e vincular-se a um território gera sentimentos de responsabilização, cuidado, acessibilidade e empatia. Organizar o serviço e trazer a população para participar de tal ação otimiza relações, valoriza, inclui e permite a esses sujeitos transformarem-se em agente de mudanças, propiciando uma cogestão comprometida na resolução de demandas do serviço de saúde.

\section{Considerações finais}

As estratégias de EPS e de Educação em Saúde adotadas na experiência constituíramse como potencializadoras na transformação das práticas do trabalho, com base em reflexões críticas, propondo o encontro entre o mundo da formação e o mundo do trabalho, a partir da interseção entre o aprender e o ensinar na realidade dos serviços.

O estudo potencializou os conhecimentos dos profissionais e da população sobre o fluxo do serviço, fazendo com que a unidade se reconstruísse e reinaugurasse não em estrutura física, mas em carga intelectual e humana na atenção, empenho ao bem-estar de ambos.

Destaca-se enquanto limitações para os momentos de EPS a demanda da unidade, o que fez com que houvesse a necessidade de desenvolver os processos formativos da equipe de enfermagem de forma separada entre técnicos e enfermeiros. 
Assim, essa experiência serve como subsídio para novos estudos e discussões que busquem qualificar o cuidado integral e autonomia de trabalho nos desafios da dimensão técnico-científica por meio da implementação do Acolhimento com Classificação de Risco.

\section{Referências}

ALMEIDA, J.R. S. et al. Educação permanente em saúde: uma estratégia para refletir sobre o processo de trabalho. Revista da ABENO, Londrina, v. 16, n. 2, p. 7-15, abr./jun. 2016. Disponível em: http://revodonto.bvsalud.org/scielo.php?script=sci_arttext\&pid=S167959542016000200003. Acesso em: $1^{\mathrm{o}}$ jun. 2020.

BRASIL. Conselho Nacional de Secretários de Saúde. A atenção primária e as redes de atenção à saúde. Brasília, 2015. Disponível em: https://www.conass.org.br/biblioteca/pdf/AAtencao-Primaria-e-as-Redes-de-Atencao-a-Saude.pdf. Acesso em: $1^{\circ}$ jun. 2020.

BRASIL. Lei $\mathbf{n}^{\mathbf{0}} \mathbf{7 . 4 9 8}$, de 25 de junho de 1986. Dispõe sobre a regulamentação do exercício da enfermagem, e dá outras providências. Disponível em:

http://www.planalto.gov.br/ccivil_03/leis/17498.htm. Acesso em: 5 set. 2020.

BRASIL. Manual de Acolhimento e Classificação de Risco. Secretaria de Saúde do Distrito Federal, 2020. Disponível em: http://www.saude.df.gov.br/wpconteudo/uploads/2017/10/MANUAL-DE-ACOLHIMENTO-ECLASSIFICA\%C3\%87\%C3\%83O-DE-RISCO-DA-REDE-SES-Web.pdf. Acesso em: $1^{\circ}$ jun. 2020.

BRASIL. Ministério da Saúde. Acolhimento a demanda espontânea: queixas mais comuns na atenção básica. v. 2. 2013. (Cadernos de Atenção Básica). Disponível em:

https://bvsms.saude.gov.br/bvs/publicacoes/acolhimento_demanda_espontanea_queixas_com uns_cab28v2.pdf. Acesso em: $1^{\text {o }}$ jun. 2020.

BRASIL. Ministério da Saúde. Portaria no 2.436, de 21 de setembro de 2017. Aprova a Política Nacional de Atenção Básica, estabelecendo a revisão de diretrizes para a organização da Atenção Básica, no âmbito do Sistema Único de Saúde (SUS). Brasília: Ministério da Saúde; 2017. Disponível em:

http://bvsms.saude.gov.br/bvs/saudelegis/gm/2017/prt2436_22_09_2017.html. Acesso em: 26 set. 2020 .

BUSNELLO, G. F. et al. Cuidados de enfermagem à puérpera na estratégia saúde da família. In: CONGRESSO SUL BRASILEIRO DE SISTEMATIZAÇÃO DA ASSISTÊNCIA DE ENFERMAGEM, 2., 2017, Chapecó. Anais [...]. Chapecó: Editora da UDESC, v. 1, p. 4042, 2017. Disponível em:

https://www.udesc.br/arquivos/ceo/id_cpmenu/1752/anais_2_CONSAI_1MICENF_15293511 791346_1752.pdf. Acesso em: 2 jun. 2020.

CARNUT, L. Cuidado, integralidade e atenção primária: articulação essencial para refletir sobre o setor saúde no Brasil. Saúde Debate, Rio de Janeiro, v. 41, n. 115, p. 1177-1186, out./dez. 2017. Doi: 10.1590/0103-1104201711515. Disponível em: 
https://www.scielo.br/pdf/sdeb/v41n115/0103-1104-sdeb-41-115-1177.pdf. Acesso em: 2 jun. 2020.

CAVALGANTE, B. L. L.; LIMA, U. T. S. Relato de experiência de uma estudante de enfermagem em um consultório especializado em tratamento de feridas. Journal of Nursing and Health, Pelotas, v. 1, n. 2, p. 94-103, jan./jun. 2012. Disponível em:

https://periodicos.ufpel.edu.br/ojs2/index.php/enfermagem/article/view/3447. Acesso em: 2 jun. 2020.

FALKENBERG, M. B. et al. Educação em saúde e educação na saúde: conceitos e implicações para a saúde coletiva. Ciênc. saúde colet., Rio de Janeiro, v. 19, n. 3, mar. 2014. Doi: 10.1590/1413-81232014193.01572013. Disponível em: https://www.scielo.br/scielo.php?script=sci_arttext\&pid=S1413$81232014000300847 \& \operatorname{lng}=$ pt\&tlng=pt. Acesso em: 2 jun. 2020.

FRANÇA, T. et al. Política de educação permanente em saúde no Brasil: a contribuição das comissões permanentes de integração ensino-serviço. Ciênc. saúde colet., Rio de Janeiro, v. 22, n. 6, jun. 2017. Doi: 10.1590/1413-81232017226.30272016. Disponível em:

https://www.scielo.br/scielo.php?pid=S1413-81232017002601817\&script=sci_arttext. Acesso em: 5 maio 2020.

HANAUER, M. C.; MADUREIRA, V. S. F. Consulta de enfermagem em domicílio: um relato de experiência do estágio supervisionado. In: CONGRESSO SUL BRASILEIRO DE SISTEMATIZAÇÃO DA ASSISTÊNCIA DE ENFERMAGEM, 2., 2017, Chapecó. Anais [...]. Chapecó: Editora da UDESC, v. 1, p. 305-307, 2017. Disponível em: https://www.udesc.br/arquivos/ceo/id_cpmenu/1752/anais_2_CONSAI_1MICENF_15293511 791346_1752.pdf. Acesso em: 01 de junho de 2020.

NEGRÃO, M. L. B. et al. The waiting room: potential for people with arterial hypertension to learn. Rev. Bras. Enferm., Brasília, v. 71, n. 6, nov./dez. 2018. Doi: 10.1590/0034-71672017-0696. Disponível em: https://www.scielo.br/scielo.php?script=sci_arttext\&pid=S0034$71672018000602930 \& l n g=e n \& t \operatorname{lng}=e n$. Acesso em: 15 maio 2020.

OPAS. Folha informativa: atenção primária à saúde. 2019. Disponível em: https://www.paho.org/bra/index.php?option=com_content $\&$ view=article\&id=5858:folhainformativa-atencao-primaria-de-saude \&Itemid=843. Acesso em: 30 maio 2020.

ROSSATO, K. et al. Acolhimento com classificação de risco na estratégia saúde da família: percepção da equipe de enfermagem. Revista de Enfermagem da UFSM, Rio Grande do Sul: Santa Maria, v. 8, n. 1, p. 144-156, jan./mar. 2018. Doi: 10.5902/2179769226655. Disponível em: https://periodicos.ufsm.br/reufsm/article/view/26655/pdf. Acesso em: 5 jun. 2020.

SILVA, A. K. L. et al. Intervenções na sala de espera: rompendo o silêncio do trabalhador. Rev. bras. saúde ocup., Rio Grande do Norte, v. 43, Supl. 1, p. 1-8, 2018. Doi: 10.1590/2317-6369000018717. Disponível em: https://www.scielo.br/pdf/rbso/v43s1/23176369-rbso-43-e4s.pdf. Acesso em: 30 maio 2020. 
SILVA, P. L. et al. Triage in an adult emergency service: patient satisfaction. Rev. esc. enferm. USP, São Paulo, v. 50, n. 3, p. 427-432, maio/jun. 2016. DOI: 10.1590/S0080623420160000400008. Disponível em:

https://www.scielo.br/scielo.php?script=sci_arttext\&pid=S0080-

62342016000300427\&lng=en\&nrm=iso. Acesso em: 5 jun. 2020.

SILVA, T. N. R. et al. Sala de espera: uma possibilidade de intervenção em Saúde do Trabalhador. Cad. Bras. Ter. Ocup., São Carlos, v. 27, n.4, out./dez. 2019. Doi: 10.4322/2526-8910.ctoRE1779. Disponível em: https://www.scielo.br/scielo.php?script=sci_arttext\&pid=S252689102019000400907\&lng=en\&nrm=iso\&tlng=pt\#B023 Acesso em: 30 maio 2020.

Submetido em 2 de junho de 2020.

Aprovado em 22 de setembro de 2020. 